\title{
Cooling Performance of Heat Sink under Partial Heating Condition
}

\author{
Youngchan Yoon, Kwan-Soo Lee \\ School of Mechanical Engineering, Hanyang University \\ 222 Wangsimni-ro, Seongdong-gu, Seoul, 04763, Korea \\ ryujifear@naver.com; ksleehy@hanyang.ac.kr
}

\section{Extended Abstract}

The cooling performance of a square-based heat sink has been analyzed with regard to the location of partial heating. A numerical model simulating forced convection was used to analyze heat transfer between the heat sink and the surrounding air. The location of the partially heated surface was continually moved from the front to the back of the heat sink while investigating the cooling performance. The results showed that the most efficient location for the partially heated surface was behind the center, when observed from the front.

Influences on optimal location of partial heating from a number of factors were analyzed. These factors are including the total heat transfer rate, inflow air velocity, the ratio of the total length of the heat sink to the width of the partially heated surface, the thermal conductivity coefficient of the heat sink and base thickness. As the width of the partially heated surface, the thermal conductivity coefficient of the heat sink, and the base thickness increased, the optimum partial heating position appeared near the center of the heat sink. Also, the larger the inflow air velocity, the farther away the optimum partial heating position was from the center, instead moving towards the rear of the heat sink. However, the magnitude of the total heat transfer rate did not affect the optimal partial heating location.

Using these results, a correlation of four factors was proposed to determine the optimal partial heating location, i.e., that which maximizes cooling performance. The correlation did not include the total heat transfer rate. It could be applied to various operating conditions. The correlation was mapped at 480 points, and validations confirmed that the correlated data had a $10 \%$ accuracy when compared to the numerical data. Using this equation, the cooling performance could be improved by up to $30 \%$ in comparison to when the partially heated surface was located only at the front end. Therefore, it is expected that the cooling performance could be improved in various electronic devices by changing the installation position of the heating element, instead of through geometric changes to the heat sink.

\section{Acknowledgements}

This work was supported by the Korea Institute of Energy Technology Evaluation and Planning (KETEP) and the Ministry of Trade, Industry \& Energy (MOTIE) of the Republic of Korea (No. 20162010103830, No. 20164010200860). 